\title{
The 'rapid filling wave' of the apex cardiogram Its relation to echocardiographic and cineangiographic measurements of ventricular filling
}

\author{
Taylor Prewitt1, Derek Gibson, David Brown', and George Sutton \\ From the Cardiac Department, Brompton Hospital, London, and The Medical Computer Centre, Westminster \\ Hospital, London
}

In order to study the relation between the 'rapid filling wave' of the apex cardiogram and left ventricular filling, simultaneous apex cardiograms, phonocardiograms, and echocardiograms were recorded in 57 patients. Continuous measurements of left ventricular dimension were derived from the echocardiograms by digitization using manual tracing and a computer. Possible errors in the use of a single dimension to assess left ventricular filling were investigated by frame-by-frame analysis of cineangiocardiograms in 37 patients, and the timing of changes in transverse diameter found to correlate closely with those in cavity area.

Mitral valve opening, shown as the initial separation of the valve cusps by echocardiography, preceded the ' $O$ ' point of the apex cardiogram in all except 3 patients, the ' $O$ ' point appearing to correlate more closely with the time of peak rate of outward wall movement. A third heart sound was present in 29 patients, and in 25 of these it occurred later than the peak rate of wall movement (mean interval $51 \mathrm{~ms}$ ). The end of rapid filling derived from the dimension trace occurred in relation to the third heart sound after a mean interval of $9 \mathrm{~ms}$, with a range from $50 \mathrm{~ms}$ before to $80 \mathrm{~ms}$ after the third sound. Peak rates of wall movement were similar in patients with and without third heart sounds.

The results show that outward left ventricular wall movement begins with a period of acceleration, with peak rates occurring synchronous with the ' $O$ ' point of the apex cardiogram and thus with the nadir of the ventricular pressure trace. Outward wall movement becomes less rapid thereafter, so that the rapid filling wave of the apex cardiogram does not reflect the time of rapid filling of the left ventricle. The ' $O$ ' point is not related to mitral valve movement nor does the third heart sound bear a consistent relation to any aspect of left ventricular wall movement.

The apex cardiogram has been widely used to study events during early diastole. Rapid retraction of the apical impulse in late systole ends with the ' $O$ ' point and is followed by an outward movement registered as a positive deflection, the 'rapid filling wave', which terminates with the ' $f$ ' point, the time of the third heart sound. The apex cardiogram at this time in the cardiac cycle thus resembles the classical ventricular filling curve (Henderson, Scarborough, and Chillingworth, 1906).

The present study was undertaken to define the relation between these features of the apex cardio-

Received 23 July 1975.

Present address: Cooper Clinic, Fort Smith, Arkansas, U.S.A. 2Present address: Brompton Hospital, Fulham Road, London S.W.3. gram and left ventricular filling, by comparison of apex cardiograms with simultaneous echocardiograms showing the time of mitral valve opening and changes in left ventricular dimension. The use of a single dimension as an index of filling was validated by comparison with changes in cavity area, using frame-by-frame analysis of cineangiocardiograms. The third heart sound, a well-known landmark in early diastole, was studied in order to gain information about its mechanism of production.

\section{Subjects and methods}

Patients

Echocardiograms and apex cardiograms suitable for detailed analysis were obtained from 57 patients, whose 
diagnoses are given in Table 1 . The diagnosis was established by cardiac catheterization or surgery in all except the 4 normal subjects. A third heart sound was present in 29 and absent in the remainder. In order to examine the relation between changes in transverse ventricular diameter and ventriculographic area, used as an index of filling, ventriculograms of 16 of these patients, and of a further 21 patients were reviewed. The diagnoses of these 37 patients are also shown in Table 1.

\section{Non-invasive techniques}

Simultaneous apex cardiograms and echocardiograms (Fig. 1 and 2) were recorded by two of the authors, with patients in the left oblique position. Echocardiograms were recorded with a Smith-Kline 20 ultrasonoscope and a Cambridge multichannel recorder, using a 2.25 $\mathrm{MHz} 1.25 \mathrm{~cm}$ probe. The probe was angled to record the echo from the anterior cusp of the mitral valve, and then directed to record echoes from both surfaces of the interventricular septum, the endocardial and epicardial

TABLE 1 Diagnosis in 57 patients studied by non-invasive techniques and in 16 of these and 21 other patients studied by cineangiocardiography

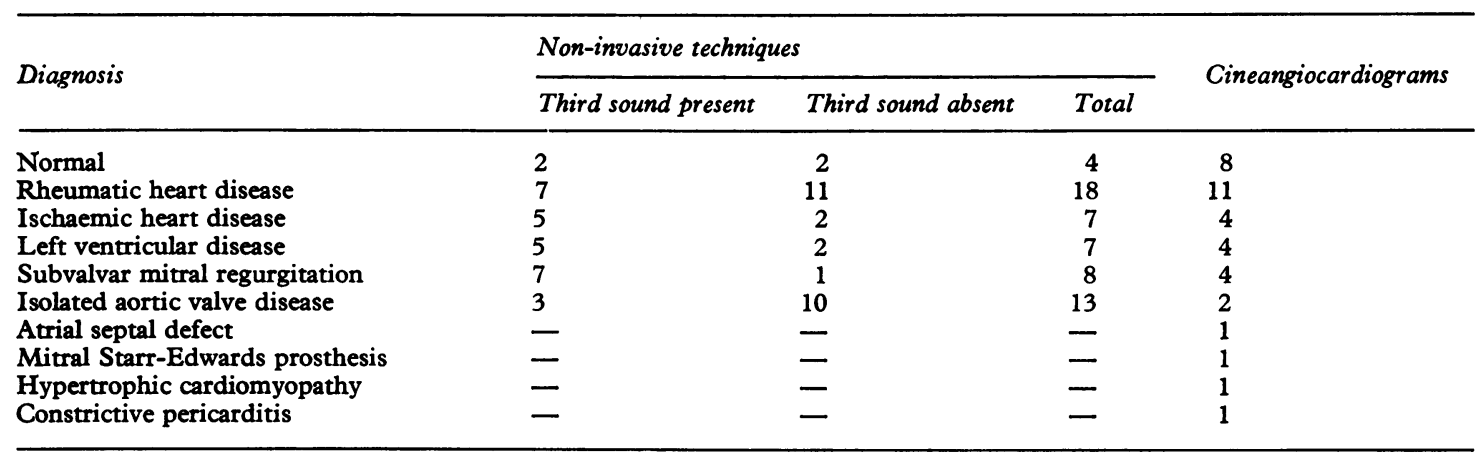

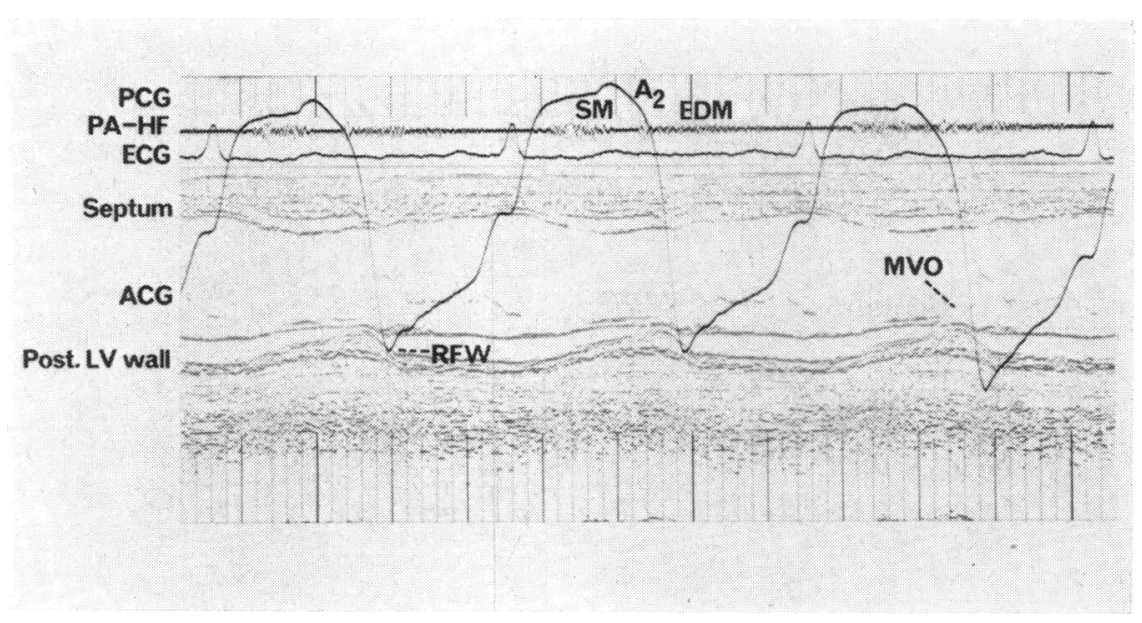

FIG. 1 Echocardiogram from a patient with aortic regurgitation with simultaneons apex cardiogram ( $A C G$ ), phonocardiogram ( $P C G)$ from putmonary area $(P A)$, and electrocardiogram (ECG). Echoes from interventricular septun, posterior wall (post $L V$ wall), and mitral valve cusps are indicated. Time markers $0.04 \mathrm{~s}$. A2=aortic valve closure, $S M=$ systolic murmur, $E D M=$ early diastolic murmur, $H F=$ high frequsncy, $R F W=$ rapid filling wave. 
surfaces of the posterior wall, and the mitral valve cusps.

Apex cardiograms were recorded with a Cambridge Scientific Instruments transducer, with a time constant of $4 \mathrm{~s}$ and a lower frequency limit of $0.04 \mathrm{~Hz}$, connected to the sensing head held at the point of maximum impulse. A simultaneous electrocardiogram was obtained, and all recordings were taken photographically at a paper speed of $100 \mathrm{~mm} / \mathrm{s}$.

Phonocardiograms were recorded from mitral and pulmonary areas with Cambridge microphones. An indirect carotid artery pulse was obtained with the Cambridge sensing device held over the right carotid artery.

\section{Angiocardiography}

Angiocardiography was performed with the patient in either the posteroanterior or the right anterior oblique position. An injection of $40-50 \mathrm{ml}$ of Triosil was made into the left ventricle at a flow rate of $25 \mathrm{ml} / \mathrm{s}$ and cine film exposed at 50 frames per s. Calibration was by means of a grid at mid-chest level.

\section{Digitization}

Echocardiograms and cineangiograms were digitized as previously described (Gibson and Brown, 1973), tracing septal and posterior wall echoes or ventriculographic outlines on to a DMAC digitizing table linked to an IBM 1800 computer. The apex cardiogram was traced in a similar manner and continous rates of change of transverse ventricular diameter were derived by digital differentiation (Fig. 3). At least 2 cardiac cycles were analysed in patients in sinus rhythm and 5 to 10 in those with atrial fibrillation. The onset of the QRS complex of the electrocardiogram was taken as zero time.

\section{Analysis of records}

The ' $O$ ' point was identified on all apex cardiograms, followed by a 'rapid filling wave' which ended at the ' $f$ ' point in all except 3 patients with mitral stenosis. Echocardiograms showed the onset of mitral valve opening as the time of initial separation of the anterior and posterior leaflets of the mitral valve (Fig. 2). The start of outward wall movement was taken as the time when the first derivative of echocardiographic dimension crossed the baseline from positive to negative at the end of systole. The time and rate of peak filling were also determined from the first derivative curve. In the patients with mitral stenosis, dimension changes were so slow and irregular that no peak values could be recognized. The 'end of rapid filling', though not a precise point, could usually be defined as a discontinuity on the first differential of the dimension curve towards middiastole (Fig. 3), and reproducible values were obtained in 48 of 57 patients.

Alternate frames from the cineangiogram of a single cardiac cycle were displayed on a Ferranti VDU, and a cursor positioned manually across the transverse diameter of the ventricle in a position below the mitral valve, approximately perpendicular to the long axis

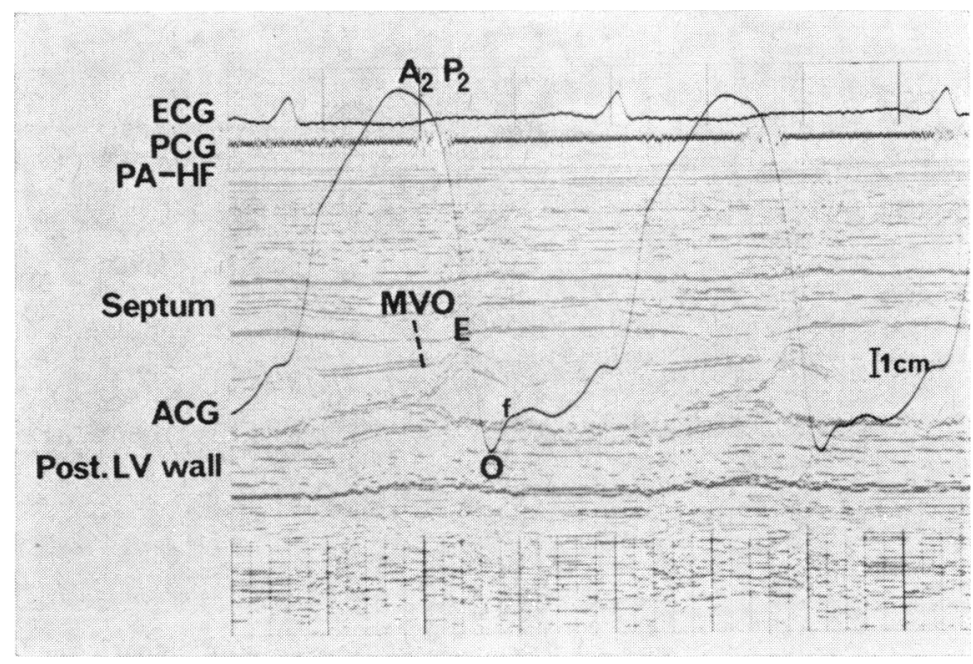

FIG. 2 Echocardiogram, phonocardiogram, and apex cardiogram from a patient with cardiomyopathy. Mitral valve opening (MVO) precedes the ' $O$ ' point of the apex cardiogram by $120 \mathrm{~ms}$, and the time of maximum separation of the valve cusps by $60 \mathrm{~ms}$. $P 2=$ pulmonary valve closure. $f=$ rapid filling wave of the apex cardiogram. 


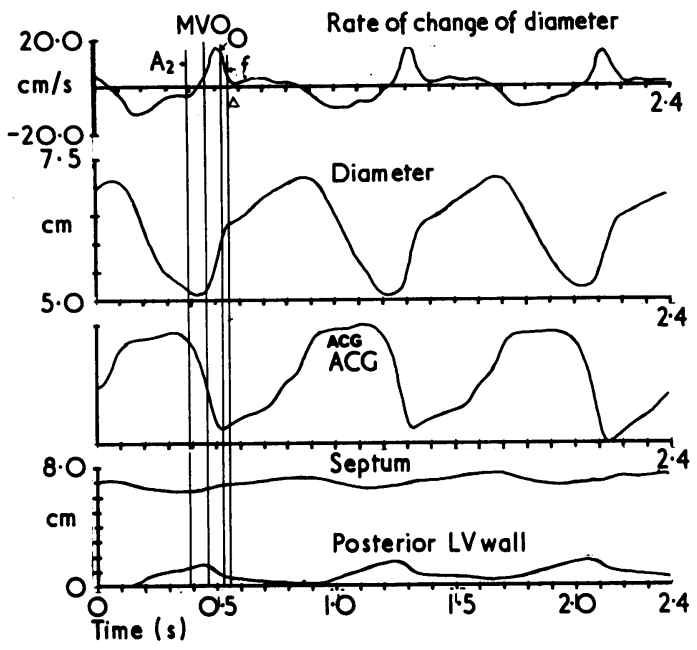

FIG. 3 Computer output of left ventricular dimension and rate of change of dimension (top two traces), apex cardiogram, and digitized records of septal and posterior wall position throughout three cardiac cycles. The times of aortic valve closure (A2) and mitral valve opening (MVO) are superimposed from the original tracing. The ' $O$ ' point of the apex cardiogram occurs shortly after the time of peak outward wall movement (shown on the top panel), and the 'f' point precedes the end of the rapid filling phase, indicated by the triangular symbol.

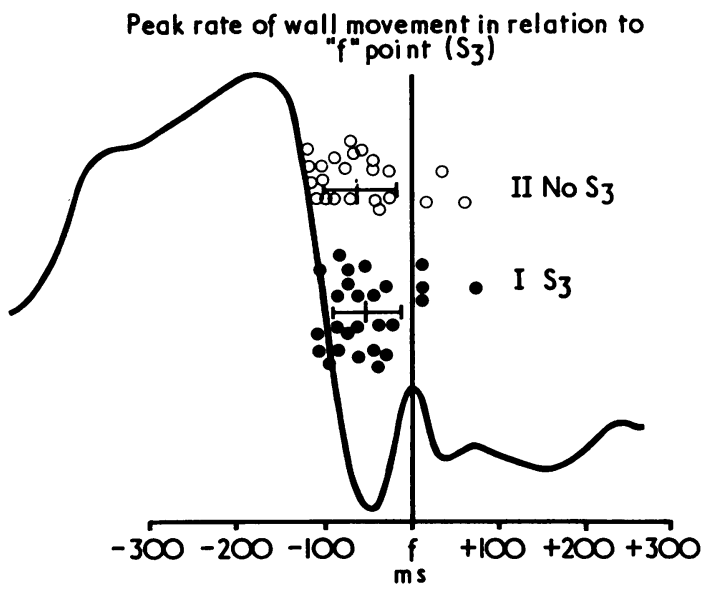

F I G. 4 Relation of peak rate of outward wall movement to the 'f' point of the apex cardiogram, for patients with and without a third heart sound (S3). Values of the time interval between the ' $f$ ' point and peak rate of wall movement from individual patients are shown superimposed on a diagram of the apex cardiogram, together with mean values and standard deviations for the two groups of patients. and the cavity outlines at the point of intersection. Changes in the length of this diameter were plotted along with those in cavity area, and the timing of the onset of outward wall movement and peak filling rate were determined from the first differential of these measurements in a similar manner to those from echocardiograms (Fig. 6).

Conventional ' $t$ ' tests were used to examine differences between means.

\section{Results}

The time of mitral valve opening, measured from the echocardiogram as the time of separation of the two cusps, preceded the ' $O$ ' point of the apex cardiogram in all but 3 of the 57 patients (mean interval $50 \mathrm{~ms}$, standard deviation $28 \mathrm{~ms}$ ). The time of maximum separation of the mitral leaflets (the ' $E$ ' point of the mitral valve echo) also preceded the ' $O$ ' point by $14 \pm 26 \mathrm{~ms}$. The ' $O$ ' point corresponded most closely in time to the peak rate of outward wall movement (mean interval $3 \pm 36 \mathrm{~ms}$ ). These results are given in detail in Table 2 .

The third heart sound followed the peak rate of outward wall movement in 25 of 29 cases, by a mean interval of $51 \pm 40 \mathrm{~ms}$ (Fig. 4). The mean value of peak rate of outward wall movement was $15 \mathrm{~cm} / \mathrm{s}$ in those patients with a third sound and $12 \mathrm{~cm} / \mathrm{s}$ in those without, a difference that was not statistically significant. The echocardiographic 'end of rapid filling' usually occurred after the ' $f$ ' point of the apex cardiogram by a mean inverval of $18 \pm 53 \mathrm{~ms}$. This interval was shorter in patients with a third

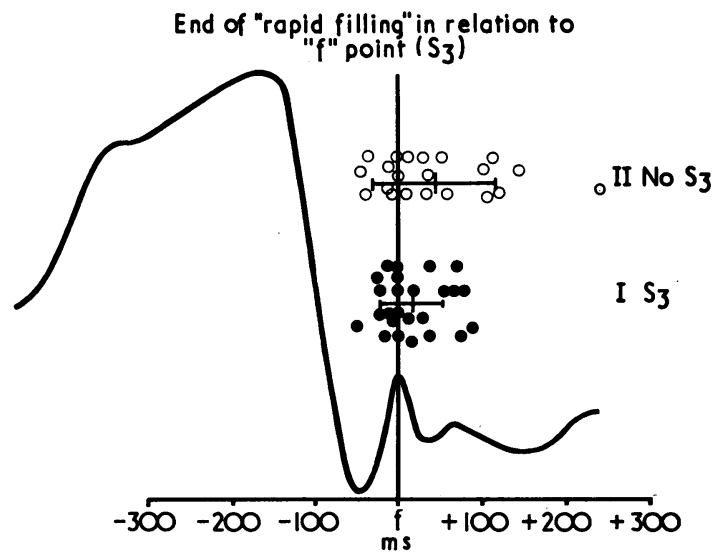

FI G. 5 Relation of 'end of rapid filling' to 'f' point of the apex cardiogram in patients with and without a third sound. The arrangement of data similar to Fig. 4. 
TABLE 2 Analysis of digitized apex cardiograms and echocardiograms in 57 patients

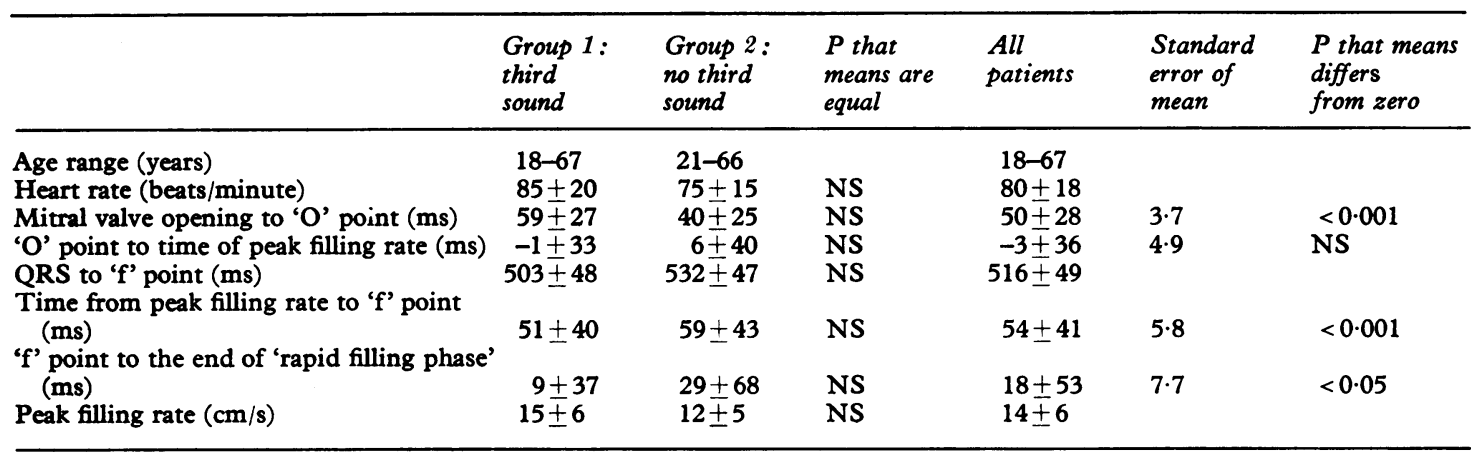

TABLE 3 Analysis of digitized cineangiograms. Comparison of measurements of transverse ventricular dimension and of ventriculographic area in 37 patients

\begin{tabular}{|c|c|c|c|}
\hline Time from onset of ventricular contraction & $\begin{array}{l}\text { Initial outward } \\
\text { movement } \\
\text { mean time (ms) } \pm S D\end{array}$ & $\begin{array}{l}\text { Peak rate of outward } \\
\text { movement } \\
\text { mean time (ms) } \pm S D\end{array}$ & $\begin{array}{l}\text { 'End of rapid } \\
\text { filling phase' } \\
\text { mean time (ms) } \pm S D\end{array}$ \\
\hline $\begin{array}{l}\text { Transverse dimension } \\
\text { Area } \\
\text { Difference } \\
\text { Standard error of mean difference } \\
P \text { that difference }=0\end{array}$ & $\begin{array}{l}287 \pm 91 \\
289 \pm 82 \\
2 \pm 31 \\
5 \\
\text { NS }\end{array}$ & $\begin{array}{l}394 \pm 91 \\
417 \pm 92 \\
23 \pm 41 \\
7 \\
<0.01\end{array}$ & $\begin{array}{l}502 \pm 118 \\
517 \pm 121 \\
15 \pm 33 \\
6 \\
<0.05\end{array}$ \\
\hline
\end{tabular}

sound $(9 \pm 53 \mathrm{~ms})$ than in those without $(29 \pm 53$ $\mathrm{ms}$ ), but the range was relatively great (Fig. 5).

Changes in transverse diameter of the left ventricle were compared with those in cavity area, both measurements being derived from digitized angiograms of the same beat (Table 3). Superimposed tracings of these two measurements used as indices of left ventricular filling were similar in each of the 37 cases (Fig. 6). Three points were identified on each tracing - the time of initial outward movement, the peak rate of outward movement, and the end of the rapid filling phase. Each of these points was usually ragistered earlier on the tracing of transverse diameter than on that in cavity area, the mean differences being $2 \pm 31,23 \pm 41$, and $15 \pm 33 \mathrm{~ms}$, respectively. The latter two differences were significantly different from zero $(P<0.01$ and $P<0 \cdot 05$, respectively).

\section{Discussion}

Some physiological events, being audible or visible, were recognized before their biological significance had been defined. The third sound is among these, having been described many years ago (Potain,
1900). Its relation to pulsations of the chest wall has been appreciated since 1909 when Thayer wrote, 'it (the third sound) was often associated with a palpable and sometimes with a visible impulse, and this impulse was shown to be identical with the normal early diastolic elevation of the apex cardiogram' (Thayer, 1909). However, though the clinical significance of the third sound and related features in the apex cardiogram have been recognized over the ensuing years, an explanation of their mechanism has remained elusive. Recent techniques have facilitated the analysis of left ventricular pressure and volume changes during the cardiac cycle, and in the present study we have related them to that early diastolic elevation of the apex cardiogram, subsequently known as the 'rapid filling wave' which starts at the ' $O$ ' point and ends with the third heart sound.

Some features of the apex cardiogram are directly related to left ventricular pressure changes. Simultaneous apex cardiograms and micromanometer recordings of left ventricular pressure in dogs have shown that the initial outward movement of the apex cardiogram coincides with the onset of the rise of ventricular pressure, that the ' $O$ ' point of the 


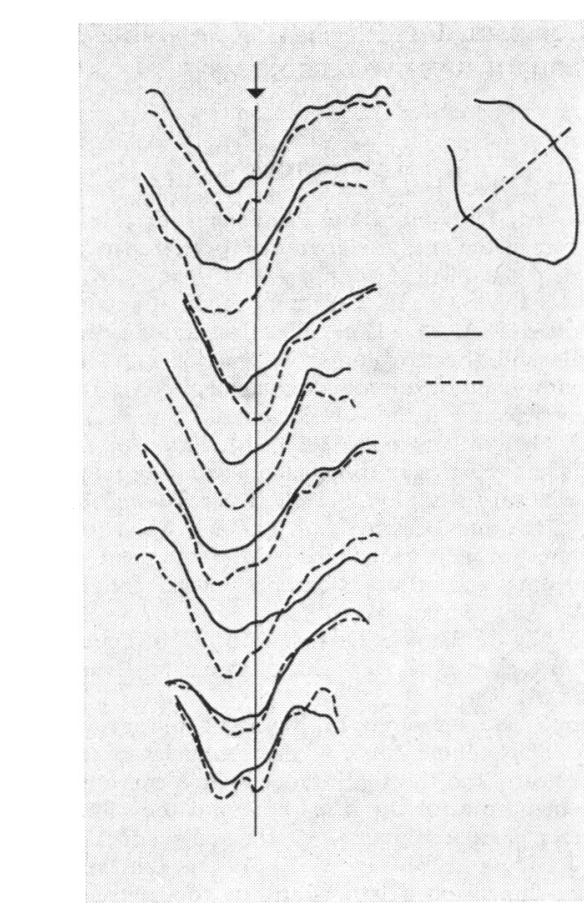

FIG 6. Changes in transverse ventricular diameter and ventricular area from 7 representative patients. Changes in area are shown by continuous, and in dimension, by dotted lines. The ventricular outline with the approximate position of the dimension studied is shown schematically on the right. The vertical line represents the time of mitral valve opening in each case.

apex cardiogram coincides with the nadir of ventricular pressure (Willems, De Geest, and Kesteloot 1971), and that the height of the ' $a$ ' wave of the apex cardiogram is related to the left ventricular enddiastolic pressure (Willems, Kestleloot, and De Geest, 1972). Similar studies in man have confirmed this relation (Bush et al., 1970). However, there is no feature of the left ventricular pressure curve that corresponds with the 'rapid filling wave' of the apex cardiogram, suggesting that factors other than left ventricular pressure are related to its genesis.

Ventricular volume changes have long been considered important in the production of the third sound (Thayer, 1908). Though the mitral subvalvar apparatus was once considered to play a fundamental role (Lewis and Dock, 1938), the demonstration of a third sound after homograft mitral and tricuspid valve replacement (Marshall and Gibson 1969) has returned attention to the interplay of haemodynamic events and ventricular wall movement. The third sound is known to occur as a physiological event in normal persons and this has been attributed to rapid ventricular filling (Thayer, 1908). Volume studies based on biplane angiograms, however, have not given unequivocal support to this idea, showing that low as well as high ventricular filling rates may be found in patients with a third heart sound (Porter et al., 1971).

The digitized echocardiogram allows non-invasive and easily repeated measurements of ventricular dimension to be recorded continuously, but a major reservation is that only a single dimension is studied, not ventricular volume. This point is particularly important in patients with ischaemic heart disease or any other condition in which incoordinate contraction occurs. Previous studies from this laboratory have compared estimates of transverse dimension and its peak rate of change made by echocardiographic and angiographic methods in patients with coherent contraction, and have shown satisfactory agreement between the two (Gibson and Brown, 1975a). In order to assess the reliability of changes in transverse diameter for timing events during filling, these were compared with changes in ventriculographic cavity area from the same beat. Changes in ventriculographic cavity area were studied because they show the timing of ventricular filling, without recourse to values of volume derived using idealizing geometrical assumptions. These studies show that there is some asynchrony between movement of the transverse diameter and filling of cavity as a whole. Outward movement of the transverse diameter usually occurs slightly ahead of the increase in ventricular area, consistent with the idea that the ventricle becomes more spherical as it enlarges in diastole (Gibson and Brown, 1975b). The mean difference in timing between the features of these two curves, however, was from 2 to $23 \mathrm{~ms}$, which can be compared with $20 \mathrm{~ms}$, the interval between successive cine frames at a speed of 50 per second. Within these limits, therefore, the transverse diameter of the left ventricle may be taken as an index of filling. Similar correlation between transverse diameter and volume changes has been reported in dogs (Horwitz and Bishop, 1972).

The ' $O$ ' point of the apex cardiogram was previously thought to coincide with mitral valve opening, since in many cases it corresponded with the opening snap in patients with mitral stenosis (Benchimol, Dimond, and Carson, 1961). More recent studies, however, have shown that this relation is a variable one (Tavel et al., 1965; Willems et al., 1971), and our observations confirm that the ' $O$ ' point coincides neither with the initial nor with maximum opening of the mitral valve leaflets 
(Fig. 2). In the present study, the ' $O$ ' point was almost always delayed with respect to mitral valve opening, even in patients with aortic regurgitation (Fig. 1), in whom early retrograde left ventricular filling presumably occurred, strongly suggesting that the apex cardiogram does not reflect volume events at this time in the cardiac cycle. Instead, the ' $O$ ' point appeared to correlate more closely with the peak rate of outward wall movement, and, taken in conjunction with observations of Willems et al. (1971) that it is also synchronous with the nadir of the ventricular pressure trace, these results are compatible with significant filling of the left ventricle as cavity pressure is falling (Porter et al., 1971). The characteristics of ventricular filling at this stage of the cardiac cycle thus differ sharply from those later in diastole, when the velocity of wall movement declines until the onset of the succeeding atrial systole.

The only haemodynamic event which showed any correlation with the third sound was the discontinuity on the dimension curve which we have termed the 'end of rapid filling'. This relation was a suggestive one in that the mean interval between the two was $9 \mathrm{~ms}$ in patients with a third sound. Exceptions to this relation, however, provoked scepticism as to its significance, in that rapid filling sometimes continued unabated through a third sound. The third sound was also unrelated to peak rates of wall movement which were high in patients with non-rheumatic mitral regurgitation and low in those with left ventricular disease. Factors other than those that we have studied in relation to ventricular filling should, therefore, be considered to explain the third sound, but we have no information as to their nature.

It is apparent that new techniques have altered traditional interpretations of the apex cardiogram in that the ' $O$ ' point is not directly related to mitral valve opening, and the 'rapid filling wave' does not reflect the time of rapid ventricular filling, while estimates of isovolumic relaxation time as the interval between aortic valve closure and the ' $O$ ' point are open to serious error. Rather than diminishing the role of the apex cardiogram, however, these observations extend its potential applications. Simultaneous echocardiograms and apex cardiograms can be complementary to one another in that pressure events can be timed with the latter, and dimension changes or valve motion studied with the former. Limitations of these techniques have been outlined but, in spite of these, much useful informa- tion can be gained with results comparable to those using standard invasive techniques.

\section{References}

Benchimol, A., Dimond, E. G., and Carson, J. C. (1961). The value of the apex cardiogram as a reference tracing in phonocardiography. American Heart fournal, 61, 485.

Bush, C. A., Lewis, R. P., Leighton, R. F., Fontana, M.E., and Weissler, A. M. (1970). Verification of systolic time intervals and the isovolumic contraction time from the apex cardiogram by micromanometer catheterization of the left ventricle and aorta. Circulation, 42, Suppl. 3, 121.

Gibson, D. G., and Brown D. (1973). Measurement of instantaneous left ventricular dimension and filling rate in man, using echocardiography. British Heart fournal, 35, 1141 .

Gibson, D. G., and Brown, D. J. (1975a). Measurement of peak rates of left ventricular wall movement in man: comparison of echocardiography with angiography. British Heart fournal, 37, 677.

Gibson, D. G., and Brown, D. J. (1975b). Continuous assessment of left ventricular shape in man. British Heart fournal, 37, 904.

Henderson, Y., Scarborough, M. M., and Chillingworth, F. P. (1906). The volume curve of the ventricles of the mammalian heart, and the significance of this curve in respect to the mechanics of the heart-beat and the filling of the ventricles. American fournal of Physiology, 16, 325.

Horwitz, L. D., and Bishop, V. S. (1972). Left ventricular pressure-dimension relationships in the conscious dog. Cardiovascular Research, 6, 163.

Lewis, J. K., and Dock, W. (1938). The origin of heart sounds and their variations in myocardial disease. fournal of the American Medical Association, 110, 271.

Marshall, J. C., and Gibson, D. G. (1969). Origin of the third heart sound. British Medical fournal, 3, 778.

Porter, C. M., Baxley, W. A., Eddleman, E. E., Frimer, M., and Rackley, C. E. (1971). Left ventricular dimensions and dynamics of filling in patients with gallop heart sounds. American fournal of Medicine, 50, 721 .

Potain, C. E. (1900). Les bruits de galop. Semaine Medicale, $20,175$.

Tavel, M. E., Campbell, R. W., Feigenbaum, H., and Steinmetz, E. F. (1965). The apex cardiogram and its relationship to haemodynamic events within the left heart. British Heart fournal, 27, 829.

Thayer, W. S. (1908). On the early diastolic heart sound (the so-called third heart sound). Boston Medical and Surgical fournal, 158, 713.

Thayer, W. S. (1909). Further observations on the third heart sound. Archives of Internal Medicine, 4, 297.

Willems, J. L., De Geest, H. D., and Kesteloot, H. (1971). On the value of apex cardiography for timing intracardiac events. American fournal of Cardiology, 28, 59.

Willems, J. L., Kesteloot, H., and De Geest, H. (1972). Influence of acute hemodynamic changes on the apex cardiogram in dogs. American fournal of Cardiology, 29, 1972.

Requests for reprints to Dr. D. G. Gibson, Cardiac Department, Brompton Hospital, Fulham Road, London SW3 6HP. 